\title{
Simple Diagnostics for Two-Way Fixed Effects
}

\author{
Pamela Jakiela*
}

March 25, 2021

\begin{abstract}
Difference-in-differences estimation is a widely used method of program evaluation. When treatment is implemented in different places at different times, researchers often use two-way fixed effects to control for location-specific and period-specific shocks. Such estimates can be severely biased when treatment effects change over time within treated units. I review the sources of this bias and propose several simple diagnostics for assessing its likely severity. I illustrate these tools through a case study of free primary education in Sub-Saharan Africa.
\end{abstract}

JEL codes: C21, O15

Keywords: difference-in-differences, program evaluation, heterogeneous treatment effects, World Development Indicators, free primary education

*Jakiela: Williams College, BREAD, CGD, IZA, and J-PAL, email: pamela.jakiela@williams.edu. I am grateful to Jessica Goldberg and Owen Ozier for helpful comments. All errors are my own. 


\section{Introduction}

Difference-in-differences estimation is a widely used method of program evaluation. Because it does not require explicit knowledge of the rule governing treatment assignment, it can be used for retrospective evaluation in a wide range of settings where pre-treatment data is available. When a treatment of interest is implemented in different places at different times, researchers often use two-way fixed effects to control for location-specific and period-specific shocks, estimating an average treatment effect across locations and time periods. Recent research demonstrates that such estimates can be severely biased - and may even be incorrectly signed - when treatment effects change over time within treated units (Goodman-Bacon 2018, De Chaisemartin and d'Haultfoeuille 2020).

I review the sources of this bias, and propose several simple diagnostics for assessing its likely severity. When a common trends assumption is satisfied, the two-way fixed effects estimator is a linear combination of the treatment effects across treated units; however, when most or all units are treated in later periods, some treated observations may receive negative weight in the calculation of the estimated treatment effect. Negative weights are a natural consequence of the two-way fixed effects specification, and are not in and of themselves a cause for concern. However, they highlight the extent to which the two-way fixed effects difference-in-differences estimation is not "model free" when treatment timing is staggered 11 Indeed, the model reflects a specific set of assumptions about the structural relationship between outcomes and treatment, and mis-specification can lead to severely biased estimates.

Though negative weighting is appropriate when treatment effects are homogeneous and the two-way fixed effects model is correctly specified, two-way fixed effects does not necessarily yield an unbiased estimate of any weighted average treatment effect when treated units

\footnotetext{
${ }^{1}$ Card and Krueger (1995) argue that the benefit of natural experiments is that they allow for "model free" evaluation of policy impacts (Card and Krueger 1995, p. 24). However, as recent analysis of differencein-differences illustrates, the assumption that analysis of natural experiments is model free is not necessarily justified once one moves away from simple comparisons of means between treated and untreated groups. See Gibbons, Suárez Serrato and Urbancic (2019) for a general discussion of mis-specification in fixed effects estimation.
} 
receive negative weight and treatment effects are heterogeneous across units (De Chaisemartin and d'Haultfoeuille 2020, Goodman-Bacon 2018, Callaway and Sant'Anna 2020, Sun and Abraham 2020, Baker, Larcker and Wang 2021). For example, if the treatment effect were zero for all units except one that received negative weight in two-way fixed effect estimation, the expected sign of the two-way fixed effects coefficient would be opposite that of any weighted average treatment effect. In general, the two-way fixed effects estimate of the treatment effect need not fall between the minimum and maximum treatment effects on treated units.

I use a simple example - the elimination of primary school fees in 15 African countries - to illustrate a set of robustness checks that can be used to address concerns about bias and identify settings where it is unlikely to be a cause for concern. Simple tests can assess the extent to which a two-way fixed effects estimator places negative weight on treated observations (because the weights are proportional to the residuals from a regression of treatment on the fixed effects), and whether the treatment effect homogeneity assumption required for such an estimator to be unbiased is likely to be appropriate (because treatment effect homogeneity implies a linear relationship between residualized outcomes and residualized treatment after removing the fixed effects). In many settings, the assumptions necessary for two-way fixed effects to provide an unbiased estimate of a weighted average treatment effect may be satisfied, and robustness checks that omit later observations can address concerns about the potential for bias.

\section{The Two-Way Fixed Effects Estimator}

I am interested in estimating the impact of treatment $D_{i t}$ on outcome $Y_{i t}$, where $i$ denotes a geographic unit of observation (e.g. country) and $t$ indicates a time period (e.g. year). I will refer to units $i$ as countries and time periods $t$ as years throughout. Treatment $D_{i t}$ varies at the country-year level; once treatment starts in country $i$, it remains "on" in all subsequent periods for that country: if $D_{i t}=1$, then $D_{i \tau}=1$ for all $\tau>t$. 
I am estimating the treatment effect of $D_{i t}$ on $Y_{i t}$ via two-way fixed effects using the regression specification:

$$
Y_{i t}=\lambda_{i}+\gamma_{t}+\beta D_{i t}+\epsilon_{i t}
$$

where $\lambda_{i}$ is a vector of country fixed effects and $\gamma_{t}$ is a vector of year fixed effects. By applying the Frisch-Waugh-Lovell theorem, we can write the OLS estimate of the treatment effect, $\beta^{\text {twfe }}$, as

$$
\beta^{t w f e}=\sum_{i t} Y_{i t}\left(\frac{\tilde{D}_{i t}}{\sum_{i t} \tilde{D}_{i t}^{2}}\right)
$$

where $\tilde{D}_{i t}$ is the residual from a regression of the treatment indicator, $D_{i t}$, on the country and year fixed effects (De Chaisemartin and d'Haultfoeuille 2020). In a balanced panel,

$$
\tilde{D}_{i t}=D_{i t}-\bar{D}_{t}-\bar{D}_{i}+\bar{D}_{\text {all }}
$$

where $\bar{D}_{t}$ is the average level of treatment across all observations in year $t, \bar{D}_{i}$ is the average level of treatment across all observations for country $i$, and $\bar{D}_{\text {all }}$ is the average level of treatment across the entire sample of country-years (Goodman-Bacon 2018).

Thus, $\beta^{\text {twfe }}$ is a weighted sum of the values of the outcome variable across all observations in the data set. When a common trends assumption holds such that country-level pretreatment means and year-level shocks are effectively differenced out by the fixed effects, $\beta^{\text {twfe }}$ is, in expectation, a linear combination of the treatment effects across country-year observations where $D_{i t}=1$. Importantly, some treated units may receive negative weight, and not all country-years are weighted equally. Intuitively, this occurs because the inclusion of two-way fixed effects transforms the binary treatment indicator $D_{i t}$ into a continuous measure of treatment intensity not explained by the fixed effects, $\tilde{D}_{i t}$. As in any univariate OLS regression of an outcome on a continuous measure of treatment intensity, observations with below mean treatment intensity receive negative weight, and may be thought of as part of the comparison group. However, in the case of two-way fixed effects, it is outcomes with below mean levels of residualized treatment intensity - after controlling for country 
and year fixed effects - that receive negative weight.

When negative weights occur among observations in the treatment group (i.e. countryyears with $D_{i t}=1$ ), they will tend to occur in early-adopter countries (where the countrylevel treatment mean is high) and in later years (when the year-level treatment mean is also high) 2 Treated country-years that receive negative weight are those where the level of treatment predicted by the country and year fixed effects exceeds one $3^{3}$ Hence, a sufficiently large never-treated group combined with enough pre-treatment data will guarantee that negative weights do not occur in the treatment group. However, in data sets with a limited number of pre-treatment periods, or with periods in which all or most units are treated, two-way fixed effects estimation will often put negative weight on the treatment effects in later periods for early-adopter units.

Negative weights on treated country-year observations are not, by themselves, a cause for concern. When treatment effects are homogeneous, the two-way fixed effects model is correctly specified because the dose response relationship between the residualized outcome variable $\tilde{Y}_{i t}$ and the residualized treatment variable $\tilde{D}_{i t}$ is linear. OLS correctly adjusts for the fact that the estimated fixed effects associated with high-treatment units and hightreatment periods are capturing some of the true treatment effect. Hence, negative weights are not a pathology, but a desired and natural consequence of the (implicit) modeling assumption we make when we difference out country and year means and estimate a single (implicitly homogeneous) treatment effect.

\footnotetext{
${ }^{2}$ As Goodman-Bacon (2018) demonstrates, when treatment timing varies across units that eventually receive treatment, $\beta^{t w f e}$ can also be decomposed into a weighted average of all possible pairwise $2 \times 2$ difference-in-differences estimators that can be constructed from the data. $\beta^{\text {twfe }}$ is a weighted average (with weights summing to one) of three types of $2 \times 2$ difference-in-differences estimators: (1) comparisons of early adopters with later adopters over periods when the later adopters are not yet treated, (2) comparisons of early adopters with later adopters over the periods when the early adopters are already treated using the early adopters as the comparison group, and (3) comparisons of ever-treated groups with the never-treated group, if there is one. The weight placed on an individual country-year observation in calculating $\beta^{\text {twfe }}$ is the sum of the weights it receives across all three types of comparisons, including those where it is used as a comparison group. While untreated country-years are never used as the treatment group in such pairwise comparisons, treated country-years are used a comparison group some of the time. Country-years that receive negative weight in the calculation of $\beta^{\text {twfe }}$ are those that are used primarily as the comparison group in the construction of pairwise $2 \times 2$ difference-in-differences estimators.

${ }^{3}$ Hence, there is a parallel with linear probability models, where predicted probabilities outside the unit interval may be viewed as an indication of mis-specification.
} 
Though negative weights are not a cause for concern when treatment effects are homogeneous, the two-way fixed effects estimator can be severely biased when treatment effects are heterogeneous - particularly when they change over time within treated units (De Chaisemartin and d'Haultfoeuille 2020, Goodman-Bacon 2018). In these cases, $\beta^{\text {twfe }}$ will not necessarily fall between the minimum and maximum treatment effects on any individual country-years $4^{4}$ For example, Baker et al. (2021) show that estimates of the impact of banking deregulation on inequality in the United States are biased because the impacts of deregulation appear to grow larger over time. Hence, it is important to test whether difference-in-differences estimates derived from two-way fixed effects estimation are influenced by the inclusion of later country-years receiving negative weight in the calculation of the average treatment effect, and - if so - whether the assumption of treatment effect homogeneity is plausible.

\section{Simple Diagnostics for Two-Way Fixed Effects}

When assessing whether difference-in-differences estimates derived from two-way fixed effects estimation are likely to be biased, it is important to answer two questions. First, do any treated units receive negative weight in the calculation of $\beta^{\text {twfe? }}$. Answering this question is straightforward since the weights are proportional to the residuals from a regression of treatment on country and year fixed effects. Second, can one reject the hypothesis that treatment effects are homogeneous? To answer this second question, one can exploit the fact that, under the assumptions of treatment effect homogeneity and common trends, the relationship between $\tilde{Y}_{i t}$ and $\tilde{D}_{i t}$ is linear; a testable implication is that the slope does not differ between the treatment group and the control group.

In what follows, I illustrate how these diagnostics can be used in practice. I present an empirical example, assessing the impact of the elimination of primary school fees in

\footnotetext{
${ }^{4}$ This is true even in cases where no treated observations receive negative weight, as the two-way fixed effects coefficient is effectively re-scaled to fit the linear relationship between outcomes and residualized treatment. However, when treatment effects are heterogeneous but are all of the same sign, the expected value of the two-way fixed effects estimator can only be signed incorrectly if treated observations receive negative weight in calculating the estimated effect.
} 
Sub-Saharan Africa on school enrollment. I use data on gross enrollment in primary and secondary school from 15 African countries that eliminated primary school fees between 1994 and 2013. These outcomes are ideal for illustrating the properties of the two-way fixed effects estimator because the elimination of primary school fees is likely to have had a large and immediate impact on primary school enrollment, but only a delayed effect on enrollment in secondary school - suggesting that the risk of bias in two-way fixed effects is larger for the latter outcome.

Data on enrollment comes from the World Bank's World Development Indicators database. My analysis includes data from 1981 through 2015. Data on the timing of the elimination of school fees comes from Koski et al. (2018). The countries included in the sample and the years that each country eliminated primary school tuition fees are listed in Online Appendix Table 1. The data set contains 15 countries, but only 13 distinct timing groups (defined as countries that eliminated school fees in the same year) since Kenya and Rwanda both eliminated primary school fees in 2003, while Benin and Lesotho both eliminated fees in 2006. All data and code used in this paper is available at https://pjakiela.github.io/TWFE.

In Online Appendix Table A2, I estimate the impact of eliminating school fees on enrollment in primary and secondary school while controlling for country and year fixed effects. Estimates suggest that introducing free primary education increased gross enrollment in primary school by 20 percentage points (p-value 0.04). These results are consistent with existing evidence from specific implementing countries demonstrating that the elimination of schools fees increased enrollment (cf. Lucas and Mbiti 2012, Njeru et al. 2014). Using the two-way fixed effects specfication, I do not find evidence that free primary education led to an increase in enrollment in secondary school (estimated coefficient -0.47 percentage points, p-value 0.88).

\subsection{Do Treated Observations Receive Negative Weight?}

Figure 1 plots the weights placed on country-year level observations in calculating the twoway fixed effects estimate of the treatment effect of eliminating primary school fees. As 
discussed above, these weights are proportional to the residuals from a regression of the treatment dummy on the set of country and year fixed effects. As expected, the weights sum to zero (across the treatment and control observations), but some treated country-year observations receive negative weight, and some untreated country-year observations receive positive weight - so the weights on country-years in the treatment group do not necessarily sum to one. Approximately 26 percent of all treated country-year observations receive negative weight in the estimation of the treatment effect $5^{5}$

Figure2 2 illustrates the distribution of negative weights across country-year observations. The unbalanced nature of the panel impacts which treated country-years receive negative weight in the estimation of the treatment effect, and a country-year may be negatively weighted when subsequent years of data from the same country are not. Nevertheless, the country-years receiving negative weight tend to be the later years of data from early-adopter countries. No treated country-year observation from before 2006 receives negative weight, and almost all observations receiving negative weight (44 of 50 negatively weighted countryyears in the case of primary enrollment and negatively weighted 33 out of 36 country-years in the case of secondary school enrollment) are concentrated among the first five countries to implement free primary schooling. This suggests two simple robustness checks that I implement in Section 3.3. (i) dropping the last years in the data set and (ii) retaining only a fixed number of post-treatment years per country. Either of these approaches can be used to identify sub-samples in which the two-way fixed effects estimator does not place negative weight on any treated country-years. However, even two-way fixed effects estimates that do not rely on negative weighting should be treated with caution when treatment effects are heterogeneous.

\footnotetext{
${ }^{5}$ Because data availability differs across country-years for the two outcome variables, the residualized treatment variable $D_{i t}$ is not identical in the two specifications (though the treatment variable is the same in both cases). In the analysis of impacts on primary school enrollment 50 out of 193 non-missing treated country-year observations receive negative weight in the calculation of the treatment effect. In the analysis of impacts on secondary school enrollment 36 out of 138 non-missing treated country-year observations receive negative weight in the calculation of the treatment effect.
} 


\subsection{Testing the Homogeneity Assumption Directly}

We have seen that the two-way fixed effects estimate of the impact of free primary education is a linear combination of country-year outcomes, and that in our sample some treated country years receive negative weight in this calculation. As discussed above, negatively weighting observations in the treatment group is appropriate if treatment effects are homogeneous, but can lead to bias when treatment effects change over time within treated units. If the homogeneity assumption holds, the relationship between the residualized outcome variable $\tilde{Y}_{i t}$ and the residualized treatment variable $\tilde{D}_{i t}$ is linear. To see this, consider a balanced panel. Let $\mu_{i}$ denote the value of the outcome variable $Y_{i t}$ in country $i$ when $t=1$, and let $\eta_{t}$ be the change in the outcome variable that would occur between period $t-1$ and $t$ in the absence of treatment (which is assumed to be constant across countries under common trends) $\mathrm{b}^{6}$ Let $\delta$ denote the homogeneous treatment effect. The value of the outcome variable for country $i$ in year $t$ can be written as

$$
Y_{i t}=\mu_{i}+\sum_{\tau=1}^{t} \eta_{t}+\delta D_{i t}
$$

and the residualized outcome variable, $\tilde{Y}_{i t}$ is equal to $\delta \tilde{D}_{i t}$. Hence, under the assumption of homogeneous treatment effects (and common trends), $\tilde{Y}_{i t}$ is a linear function of $\tilde{D}_{i t}$, and the slope does not differ between the treatment group and the comparison group.

Figure 3 presents a scatter plot of these residuals; Panel A shows residuals from the regressions using primary school enrollment as the outcome of interest, while Panel B shows the residuals from analysis using secondary school enrollment as the outcome. In both cases, local polynomial regressions suggest that the relationship between $\tilde{Y}_{i t}$ and $\tilde{D}_{i t}$ may not be perfectly linear, particularly near the extremes of the support. However, in the case of primary school enrollment (Panel A), there is no obvious evidence that the slope differs between the treatment group and the comparison group. OLS regression analysis of $\tilde{Y}_{i t}$ on $\tilde{D}_{i t}$ confirms this (Online Appendix Table A3): the slope of the estimated linear relationship

\footnotetext{
${ }^{6}$ Hence, $\eta_{1}=0$
} 
between $\tilde{Y}_{i t}$ and $\tilde{D}_{i t}$ does not differ between the treatment group and the comparison group (p-value 0.20). The situation is different when we look at the impacts of free primary on secondary school enrollment: there is clear evidence that the slope of the relationship between the residualized outcome variable $\tilde{Y}_{i t}$ and the residualized treatment variable $\tilde{D}_{i t}$ is not the same for the treatment and comparison group. Regression analysis confirms this: the slope of the relationship between $\tilde{Y}_{i t}$ and $\tilde{D}_{i t}$ is significantly higher for treated countryyears than for comparison observations (Online Appendix Table A3, p-value 0.01). Hence, the relationship between the elimination of primary school fees and gross enrollment in secondary school is not consistent with the assumptions required for two-way fixed effects estimation.

\subsection{Robustness Checks}

Under the assumption of homogeneous treatment effects, dropping some treated countryyears from the data set should not affect the expected value of the estimated treatment effect (assuming common trends). Hence, robustness checks of this type, include jackknife estimation, can be viewed as further tests of the assumption of treatment effect homogeneity. I present several examples appropriate to difference-in-differences settings below.

Figure 4 presents specifications that omit later years from the data set. For primary school enrollment (Panel A of Figure 4), the estimated treatment effect declines as the last year included in the analysis increases from 2000 to 2004, though no country-years in the treatment group are negatively weighted in calculating these treatment effect - suggesting treatment effect heterogeneity either across countries (earlier vs. later adopters) or over time (in the first few years after treatment vs. later years, among early-adopter countries). As the last included year increases from 2005 to 2015, the proportion of treatment negatively weighted country-year observations increases from three percent in 2005 to 26 percent in 2015. However, the estimated treatment effect remains remarkably stable across these specifications - suggesting that the negatively weighted observations are not driving the results. When we examine impacts on secondary school enrollment (Panel B of Figure 4), 
the estimated treatment effect appears quite stable across specifications - though we know that the linear relationship between residualized outcomes and residualized treatment does not hold in this case.

Online Appendix Figure A1 presents a related robustness check: varying the number of years of post-treatment data retained for each country. In this case, the estimated impact on primary enrollment is quite stable across specifications, but the estimated impact on secondary enrollment increases as more post-treatment years are included in the analysis. The stability in the estimated impact on primary enrollment suggests that heterogeneity apparent in Figure 4 resulted from unusually large impacts in the first countries to implement free primary education rather than changes in treatment effects over time within each country - possibly because the first countries to implement free primary education had lower pre-FPE levels of gross enrollment 7

In Online Appendix Figure A2, we implement a final robustness check: dropping individual countries from the analysis. Again, there is suggestive evidence of some treatment effect heterogeneity, though we can never reject the equality of the estimated coefficients across specifications. All specifications suggest that eliminating primary school fees increased gross primary enrollment (Panel A of Online Appendix Figure A2), though the estimated treatment effect is slightly smaller when Malawi or Uganda (two of the earliest adopters) are excluded - consistent with the evidence discussed above. The estimated impacts on secondary school enrollment appear quite robust unless Malawi (the first country in the data set to eliminate primary school fees) is excluded. In both cases, the evidence suggests that the assumption of treatment effect heterogeneity should be treated with caution.

\footnotetext{
${ }^{7}$ The four countries that implemented free primary education in the 1990s - Malawi, Ethiopia, Ghana, and Uganda - had substantially lower gross enrollment in the 1980s (with an average gross enrollment ratio of 61.1 ) than the 11 countries that implemented free primary in 2000 or later (those countries had an average gross enrollment ratio of 79.3 in the $1980 \mathrm{~s}$ ).
} 


\section{Conclusion}

In this paper, we use a case study of free primary education in Sub-Saharan Africa to illustrate a set of simple diagnostics and robustness checks that can be used to assess the potential for bias in two-way fixed effects. When a common trends assumption holds, the two-way fixed effects estimator is a linear combination of outcomes in treated country-years, but in some cases a subset of treated country-years are negatively weighted. One can test for the extent of negative weighting, since the weights are proportional to the residuals from a regression of treatment on country and year fixed effects.

Negative weights are not a problem when treatment effects are homogeneous; in fact, they are a natural consequence of the (implicit) assumption of treatment effect homogeneity in two-way fixed effects. When the assumption of homogeneous treatment effects is valid, the residuals from a regression of the outcome variable on country and year fixed effects are linearly related to the residuals from a regression of treatment on country and year fixed effects - and the slope of this linear relationship does not differ between the treatment group and the comparison group.

When negative weights are present or there is evidence of treatment effect heterogeneity, a range of alternative estimators are available (cf. Callaway and Sant'Anna 2020, Sun and Abraham 2020) 8 However, these estimators may have less statistical power than the pooled estimator, so it may be preferable to use traditional two-way fixed effects when the underlying identification assumptions seem plausible. I have proposed a simple test of treatment effect heterogeneity, but researchers should be alert to the possibility that the test could lack sufficient power in their data or context. Statistical reasoning should be combined with introspection in assessing whether the assumptions required for two-way fixed effects estimation seem appropriate to the setting.

Even when no treated country-years receive negative weight, the two-way fixed effects estimator does not place equal weight on all treated country-years and imposes a linear dose-

\footnotetext{
${ }^{8}$ See Baker et al. (2021) for an accessible introduction to the estimators proposed by (Callaway and Sant'Anna 2020) and (Sun and Abraham 2020).
} 
response relationship that may be mis-specified (Baker et al. 2021, Sun and Abraham 2020). When impacts are heterogeneous, it is not clear that applied researchers will wish to weight early-adopters more heavily than later-adopters - as would happen if all treated countryyears received equal weight. When treatment effects are not homogeneous, many different policy-relevant average treatment effects may exist. Researchers may prefer to characterize the extent and nature of heterogeneity - for example, through jackknife estimation and other forms of sensitivity analysis - rather than focusing on a single average treatment effect that may or may not be policy relevant or externally valid, depending on the context. As the range of difference-in-differences related approaches expands, researchers have more opportunity to choose a method that identifies their estimand of interest.

Difference-in-differences estimation through two-way fixed effects is one of the most widely used approaches for evaluating social policy. Recent research highlights the potential pitfalls of two-way fixed effects, but these criticisms should not be interpreted as an indication that two-way fixed effects is no longer a credible identification strategy. Instead, these criticisms and the newly-developed tools that have emerged in parallel provide an analytical framework for more nuanced and better understood difference-in-differences estimation via two-way fixed effects. 


\section{References}

Baker, Andrew, David F. Larcker, and Charles C.Y. Wang, "How Much Should We Trust Staggered Difference-In-Differences Estimates?," Finance Working Paperr 7361, European Corporate Governance Institute 2021.

Callaway, Brantly and Pedro H.C. Sant'Anna, "Difference-in-Differences with Multiple Time Periods," Journal of Econometrics, 2020.

Card, David and Alan B. Krueger, Myth and Measurement: The New Economics of the Minimum Wage, Princeton University Press, 1995.

Chaisemartin, Clément De and Xavier d'Haultfoeuille, "Two-Way Fixed Effects Estimators with Heterogeneous Treatment Effects," American Economic Review, 2020, 110 (9), 2964-96.

Gibbons, Charles E., Juan Carlos Suárez Serrato, and Michael B. Urbancic, "Broken or fixed effects?," Journal of Econometric Methods, 2019, 8 (1), 1-12.

Goodman-Bacon, Andrew, "Difference-in-Differences with Variation in Treatment Timing," Working Paper 25018, National Bureau of Economic Research 2018.

Lucas, Adrienne M and Isaac M Mbiti, "Does Free Primary Education Narrow Dender Differences in Schooling? Evidence from Kenya," Journal of African Economies, 2012, $21(5), 691-722$.

Njeru, Wilfred K., Moses N. Muiruri, George M. Njeru, and Ruth Thinguri, "An Investigation on Impact of Free Primary Education on Quality of Education in Kenya Primary Schools," Journal of Education and Practice, 2014, 5 (20), 26-31.

Strumpf, Erin C. Koski Alissaand, Jay S. Kaufman, John Frank, Jody Heymann, and Arijit Nandi, "The Impact of Eliminating Primary School Tuition Fees on Child Marriage in Sub-Saharan Africa: A Quasi-Experimental Evaluation of Policy Changes in 8 Countries," PLOS one, 2018, 13 (5), e0197928.

Sun, Liyang and Sarah Abraham, "Estimating Dynamic Treatment Effects in Event Studies with Heterogeneous Treatment Effects," Journal of Econometrics, 2020. 
Figure 1: Two-Way Fixed Effects Weights, by Treatment Status

Panel A: Dependent Variable: Gross Enrollment in Primary School

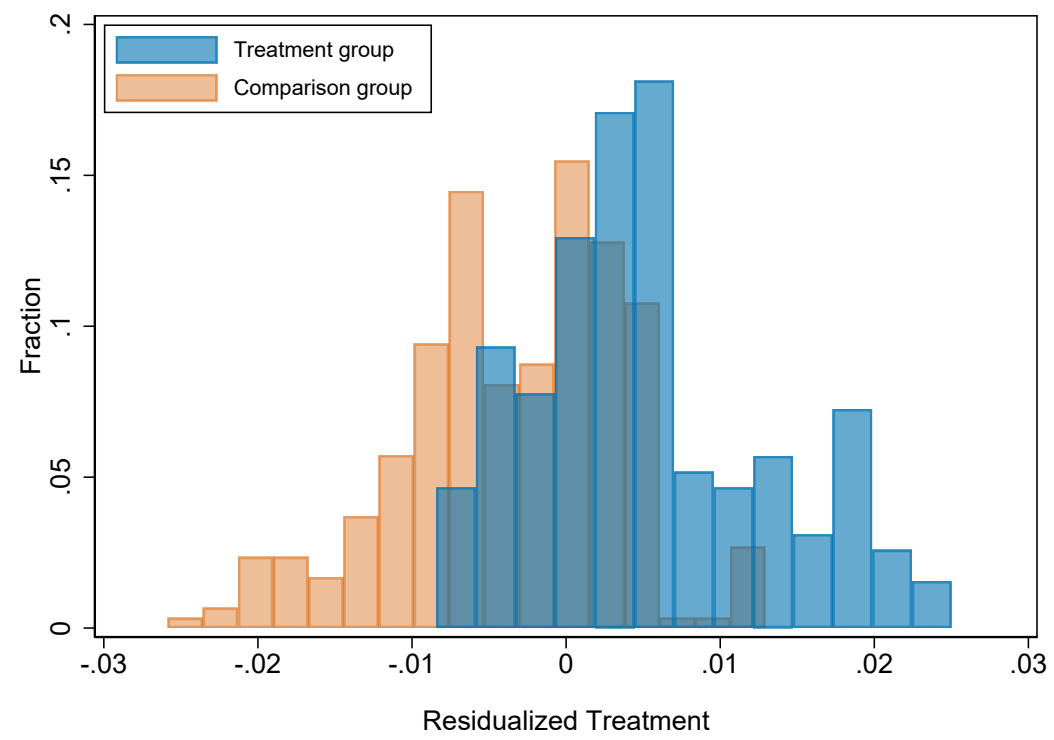

Panel B: Dependent Variable: Gross Enrollment in Secondary School

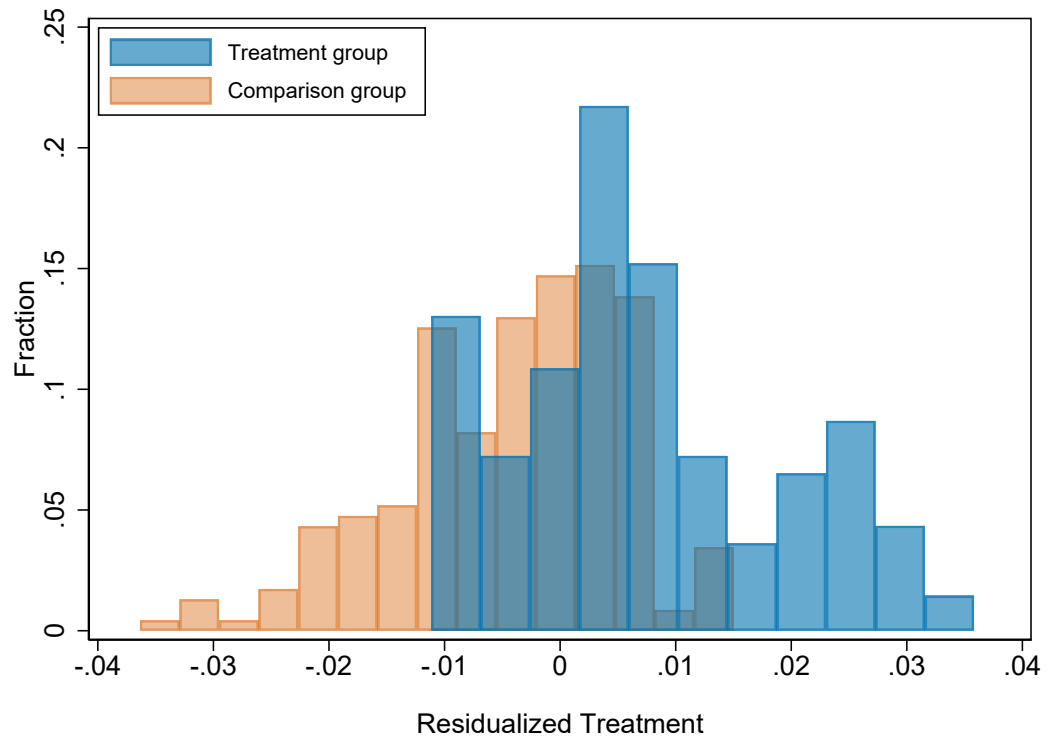

The figure presents histograms of the weights used to calculate the two-way fixed effects estimates of the impact of eliminated primary school fees on gross enrollment in primary school (Panel A) and secondary school (Panel B). The weights are the residuals from a regression of treatment on country and year fixed effects, scaled by the sum of the squared residuals across all observations. See De Chaisemartin and d'Haultfoeuille (2020) for discussion. The weights are not identical in the two specifications because both panels are imbalanced, but the missing country-years differ across the two outcome variables. 
Figure 2: Weights Used in Two-Way Fixed Effects, by Country and Year

Panel A: Dependent Variable: Gross Enrollment in Primary School
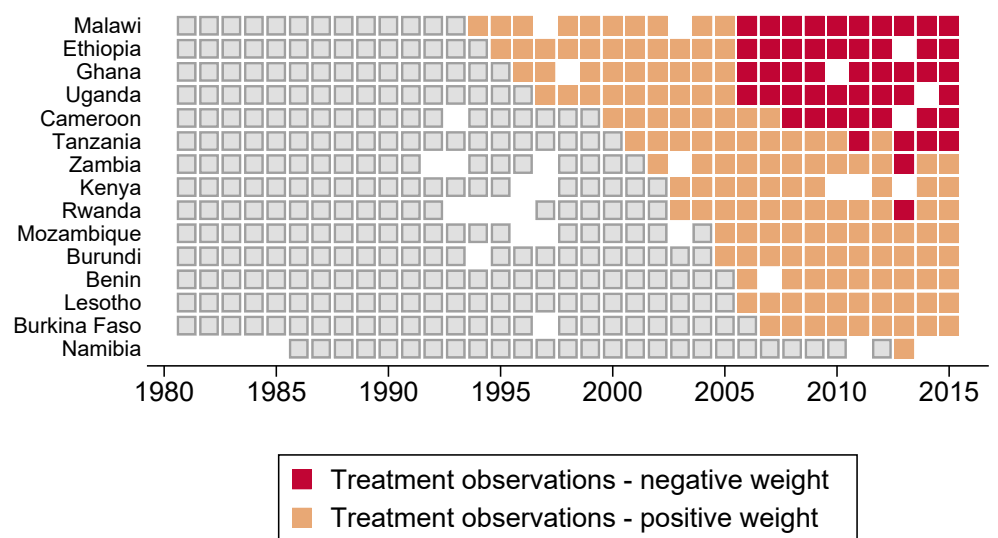

Panel B: Dependent Variable: Gross Enrollment in Secondary School
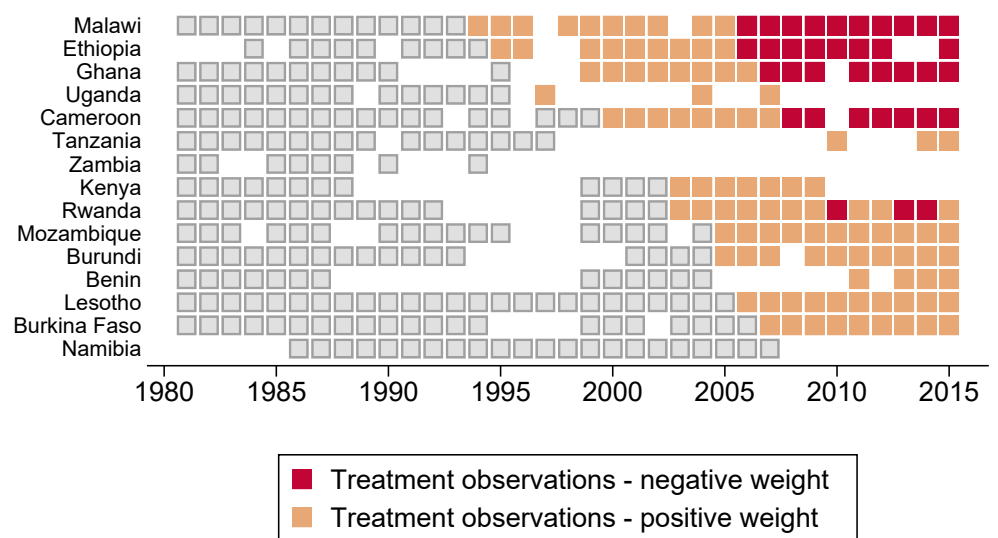

The figure characterizes the weights used to calculate the two-way fixed effects estimates of the impact of eliminated primary school fees on gross enrollment in primary school (Panel A) and secondary school (Panel B). The weights are the residuals from a regression of treatment on country and year fixed effects, scaled by the sum of the squared residuals across all observations. See De Chaisemartin and d'Haultfoeuille (2020) for discussion. The weights are not identical in the two specifications because both panels are imbalanced, but the missing country-years differ across the two outcome variables (as shown in the figure). 
Figure 3: The Association Between Residualized Outcomes and Residualized Treatment

Panel A: Dependent Variable: Gross Enrollment in Primary School

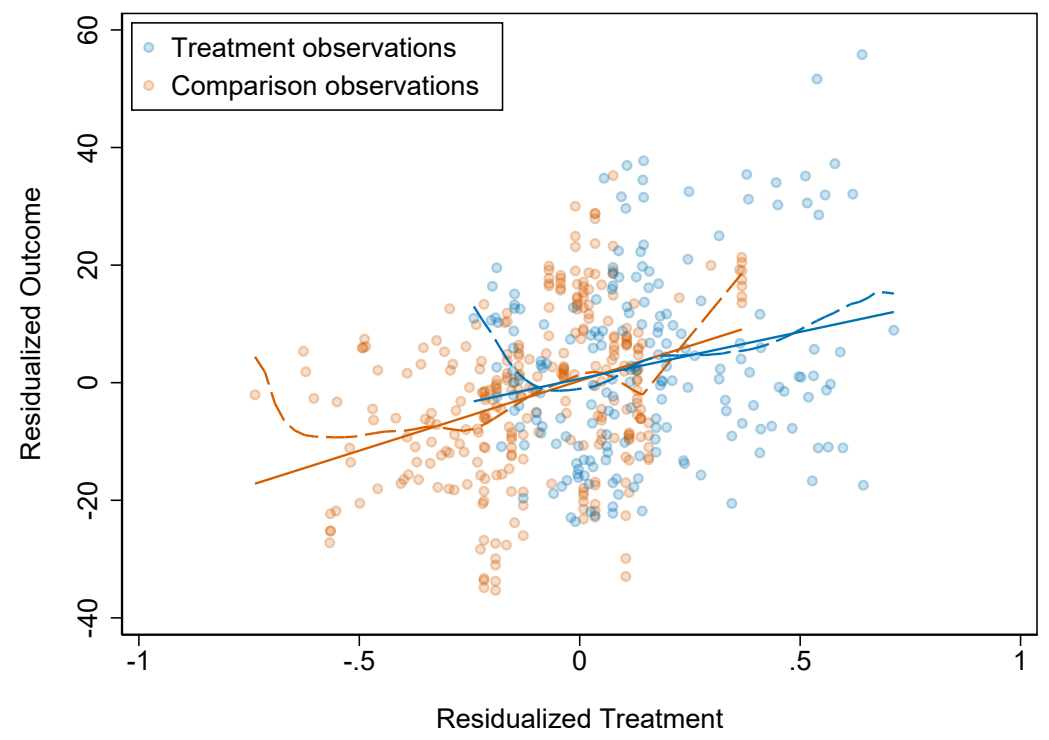

Panel B: Dependent Variable: Gross Enrollment in Secondary School

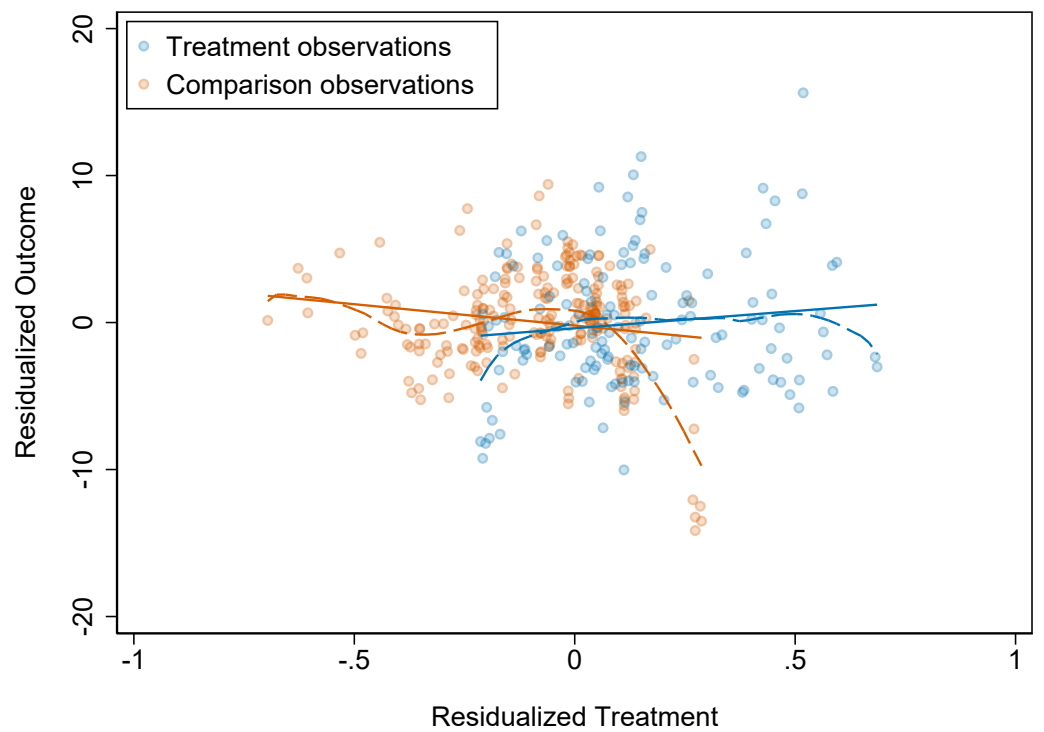

The figure plots the relationship between the residuals from a regression of the outcome variable (gross enrollment) on country and year fixed effects and the residuals from a regression of treatment on country and year fixed effects. If treatment effects are homogeneous, the relationship is linear. The line of best fit and a local linear regression of residuals from the control group appears in orange; the line of best fit and a local linear regression of residuals from the treatment group appears in blue. 
Figure 4: Robustness to Exclusion of Later Years in Data Set

Panel A: Dependent Variable: Gross Enrollment in Primary School

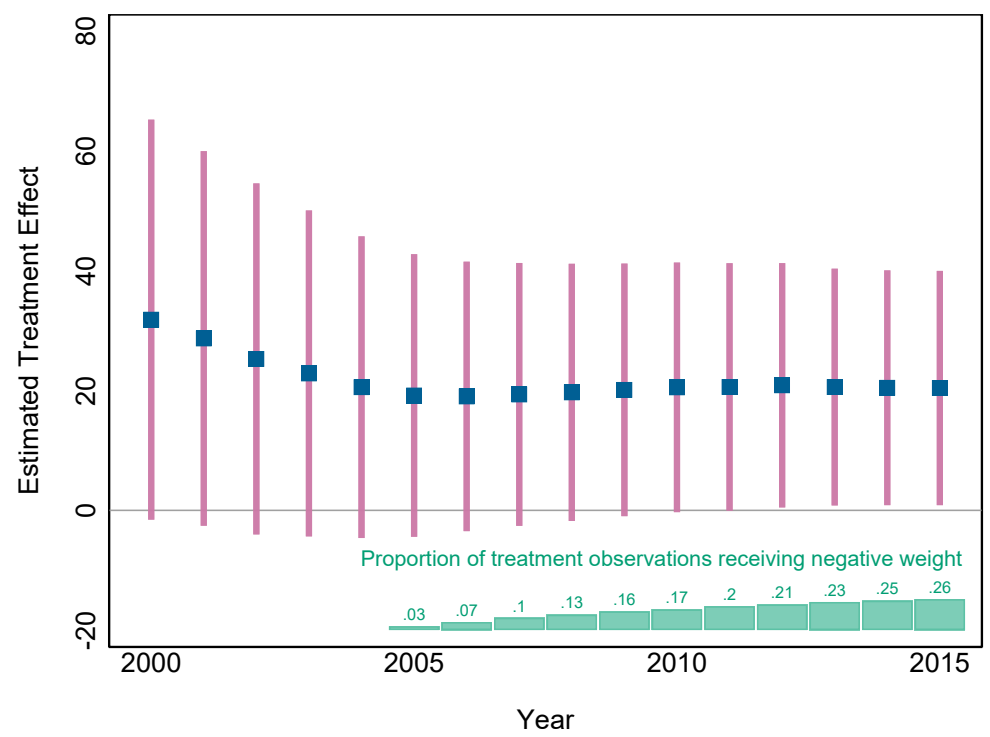

Panel B: Dependent Variable: Gross Enrollment in Secondary School

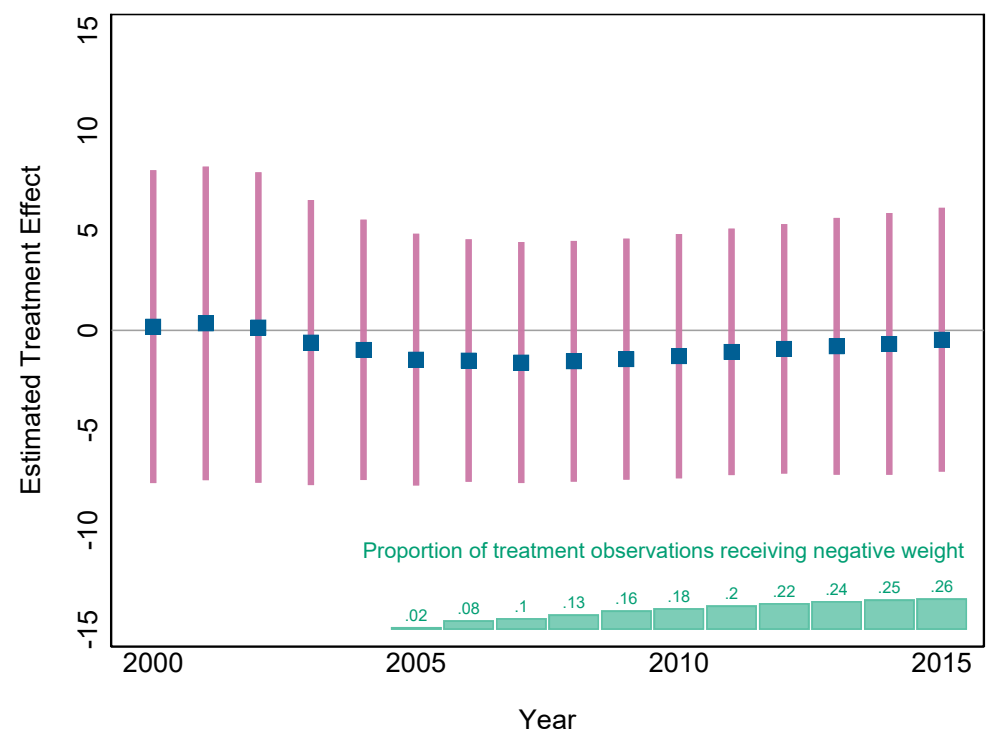

The figure summarizes the estimated two-way fixed effects coefficients and the associated confidence intervals from regressions of gross enrollment in primary school (Panel A) and gross enrollment in secondary school (Panel B) on the indicator for free primary education. Each coefficient represents a different regression that includes data from 1981 through the year indicated on the $x$-axis. Green bars indicate the proportion of treatment country-years that are negatively weighted in the calculation of the two-way fixed effects coefficient. 


\section{A Online Appendix: not for print publication}

Table A1: Countries Included in the Analysis

\begin{tabular}{lr}
\hline \hline CountRY & FPE \\
\hline Benin & 2006 \\
Burkina Faso & 2007 \\
Burundi & 2005 \\
Cameroon & 2000 \\
Ethiopia & 1995 \\
Ghana & 1996 \\
Kenya & 2003 \\
Lesotho & 2006 \\
Malawi & 1994 \\
Mozambique & 2005 \\
Namibia & 2013 \\
Rwanda & 2003 \\
Tanzania & 2001 \\
Uganda & 1997 \\
Zambia & 2002 \\
\hline FPE indicates & the year \\
free primary & education \\
was introduced & as na- \\
tional policy. & Data on \\
the introduction & of free \\
primary education comes & from Koski et al. (2018).
\end{tabular}


Table A2: Difference-in-Differences Estimates of Impacts of Free Primary Education

\begin{tabular}{lcc}
\hline \hline & $\begin{array}{c}\text { Dependent Variable: } \\
\text { Primary SCHOOL }\end{array}$ & SECrollment in... \\
& $(1)$ & $(2)$ \\
\hline Free primary education & 20.43 & -0.47 \\
& $(9.12)$ & $(3.08)$ \\
& {$[0.04]$} & {$[0.88]$} \\
Country fixed effects & Yes & Yes \\
Year fixed effects & Yes & Yes \\
\hline $\begin{array}{l}\text { Dependent variable: gross enrollment ratio. Data on gross enrollment ratio in 15 countries } \\
\text { comes from the World Development Indicators, years 1981 through 2015. Standard errors } \\
\text { (clustered at the country level) in parentheses; p-values in brackets. }\end{array}$
\end{tabular}


Table A3: Testing Relationship Between Residualized Outcomes, Residualized Treatment

\begin{tabular}{lcc}
\hline \hline & \multicolumn{2}{c}{ TWFE Dep. Var.: Gross Enrollment in... } \\
& Primary School & SECondary School \\
& $(1)$ & $(2)$ \\
\hline Residualized treatment & 23.76 & -2.90 \\
& $(3.97)$ & $(1.36)$ \\
Treatment group & {$[0.00]$} & {$[0.03]$} \\
& 0.34 & -0.19 \\
Treatment group $\times$ residualized treatment & $(1.51)$ & $(0.47)$ \\
& {$[0.82]$} & {$[0.69]$} \\
& -7.81 & 5.25 \\
& $(6.07)$ & $(1.99)$ \\
\hline
\end{tabular}

Dependent variable is the residual from a regression of the gross enrollment ratio on country and year fixed effects. Residualized treatment is the residual from a regression of the treatment dummy (an indicator for years when primary education was free) on country and year fixed effects. Data on gross enrollment ratio in 15 countries comes from the World Development Indicators, years 1981 through 2015. Standard errors in parentheses; p-values in brackets. 
Figure A1: Robustness to Exclusion of Later Post-Treatment Years

Panel A: Dependent Variable: Gross Enrollment in Primary School

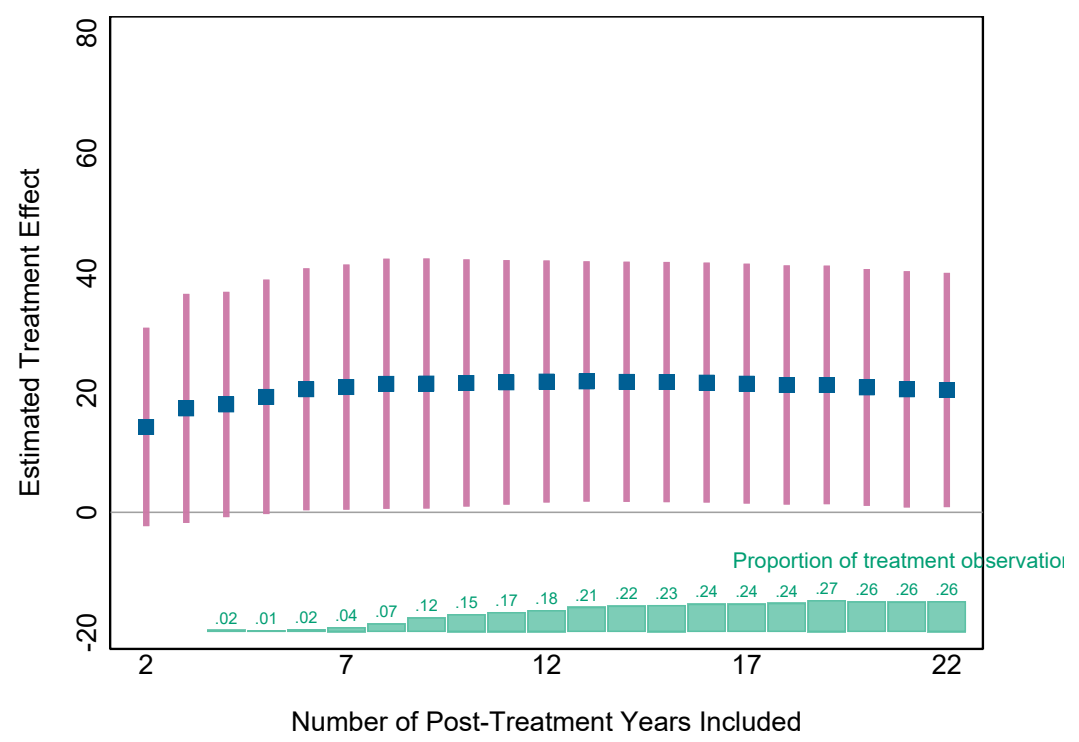

Panel B: Dependent Variable: Gross Enrollment in Secondary School

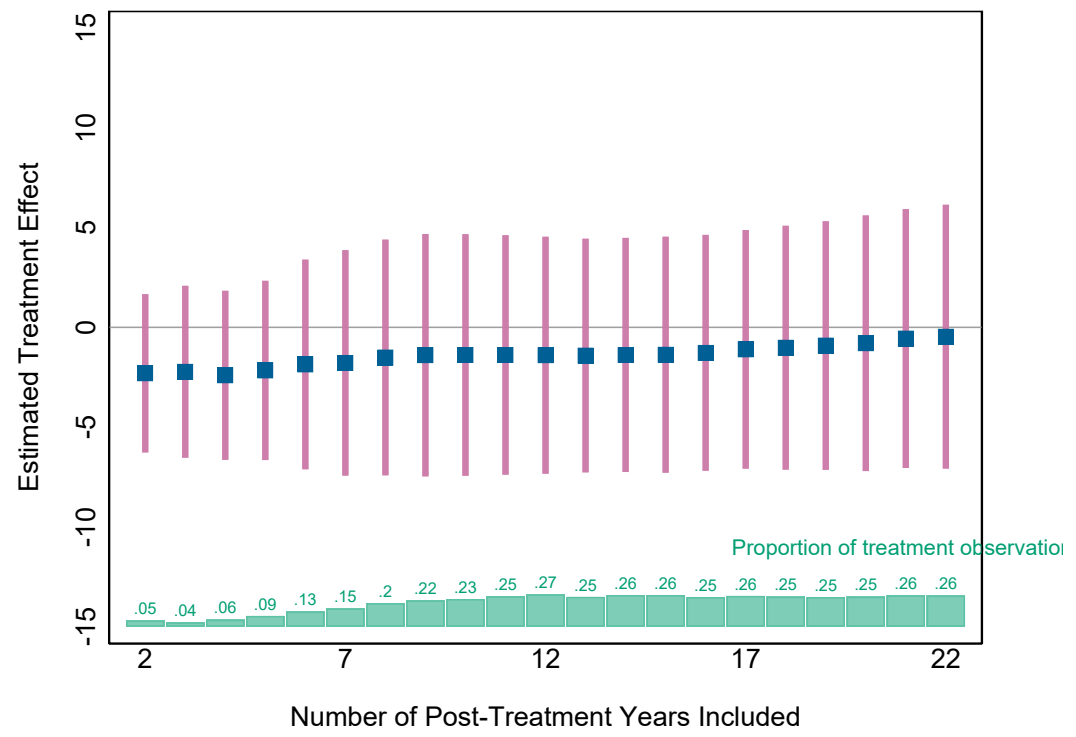

The figure summarizes the estimated two-way fixed effects coefficients and the associated confidence intervals from regressions of gross enrollment in primary school (Panel A) and gross enrollment in secondary school (Panel B) on the indicator for free primary education. Each coefficient represents a different regression that includes data from 1981 through the year $x$ years after the introduction of free primary education (within a given country). Green bars indicate the proportion of treatment country-years that are negatively weighted in the calculation of the two-way fixed effects coefficient. 
Figure A2: Robustness to Exclusion of Individual Countries

Panel A: Dependent Variable: Gross Enrollment in Primary School

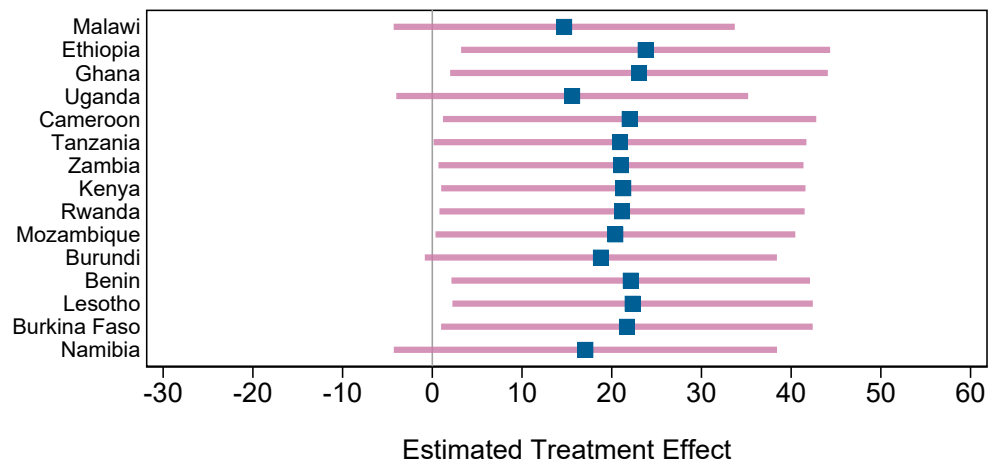

Panel B: Dependent Variable: Gross Enrollment in Secondary School

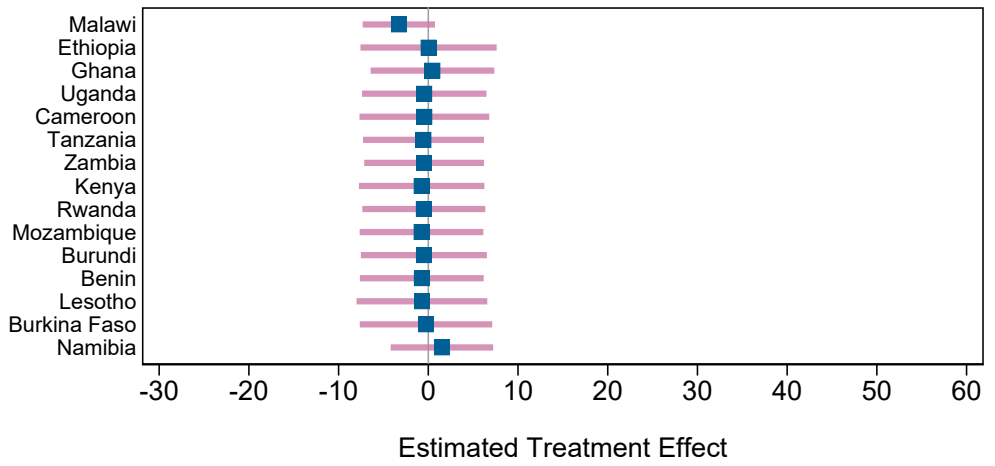

The figure summarizes the estimated two-way fixed effects coefficients and the associated confidence intervals from regressions of gross enrollment in primary school (Panel A) and gross enrollment in secondary school (Panel B) on the indicator for free primary education. Each coefficient represents a different regression that omits the country indicated on the $y$-axis. 